\title{
Deterministic and Stochastic Study for an Infected Computer Network Model Powered by a System of Antivirus Programs
}

\author{
Youness El Ansari, Ali El Myr, and Lahcen Omari \\ Laboratory of Computer Sciences, Modeling and Systems, Department of Mathematics, Faculty of Sciences, \\ Sidi Mohamed Ben Abdellah University, Dhar-Mahraz, BP 1796, Atlas, Fez, Morocco
}

Correspondence should be addressed to Youness El Ansari; y.elansari4@gmail.com

Received 13 June 2017; Revised 29 August 2017; Accepted 5 September 2017; Published 18 October 2017

Academic Editor: Yong Zhou

Copyright (C) 2017 Youness El Ansari et al. This is an open access article distributed under the Creative Commons Attribution License, which permits unrestricted use, distribution, and reproduction in any medium, provided the original work is properly cited.

\begin{abstract}
We investigate the various conditions that control the extinction and stability of a nonlinear mathematical spread model with stochastic perturbations. This model describes the spread of viruses into an infected computer network which is powered by a system of antivirus software. The system is analyzed by using the stability theory of stochastic differential equations and the computer simulations. First, we study the global stability of the virus-free equilibrium state and the virus-epidemic equilibrium state. Furthermore, we use the Itô formula and some other theoretical theorems of stochastic differential equation to discuss the extinction and the stationary distribution of our system. The analysis gives a sufficient condition for the infection to be extinct (i.e., the number of viruses tends exponentially to zero). The ergodicity of the solution and the stationary distribution can be obtained if the basic reproduction number $\mathscr{R}_{p}$ is bigger than 1 , and the intensities of stochastic fluctuations are small enough. Numerical simulations are carried out to illustrate the theoretical results.
\end{abstract}

\section{Introduction}

A computer virus is a small malicious program that spreads from host to host and has a great ability to replicate itself into or over data files during the execution of programs. On a computer network, the interconnectivity of workstations makes the spread of viruses easier. In fact, an infected computer can spread viruses (malware) through the other connected nodes, which causes severe damage like reformatting the hard drive, unexplained data loss, slowing computer performance, corrupting databases, and so forth. Consequently, mathematics specialists are becoming more aware of the importance of protecting systems. To clean and protect the network, an antivirus is a computer program designed to identify and erase malicious software in the system.

Similar to the biological virus systems, many mathematical models have been proposed to describe quantitatively the spread of infections into a computer network [1-9]. Following a deterministic approach, Mishra and Jha [1] have formulated and analyzed the effect of quarantine on recovered nodes. Yuan and Chen [2] discussed an $e$-SEIR network virusepidemic model using the theory of stability in differential equations. Shukla et al. [3] modeled and studied the effect of antivirus software on infected computer network; they proved that, under certain conditions, the used antivirus can successfully clean the system.

Other authors have followed the stochastic approach to describe the spread of viruses in a computer network [4, 5]. Zhang et al. [4] proposed and investigated a stochastic computer virus spread model. By constructing a suitable Lyapunov function, they established the necessary conditions for the virus-free equilibrium and viral equilibrium to be stabilized.

To study the effect of antivirus software on its ability to clean an infected computer network, Shukla et al. [3] have proposed the following deterministic system:

$$
\begin{aligned}
d X & =\left(A-\beta X Y-d X-k X+\pi Y V_{a}\right) d t \\
d Y & =\left(\beta X Y-d Y-\alpha Y-\pi Y V_{a}\right) d t \\
d Z & =(k X-d Z) d t \\
d V_{a} & =\left(\mu Y-\mu_{0}\left(V_{a}-V_{a 0}\right)\right) d t
\end{aligned}
$$


here $X(t)$ denotes the number of susceptible nodes at time $t \geq 0, Y(t)$ the number of infected nodes, $Z(t)$ the number of protected nodes, and $V_{a}(t)$ the number of antivirus programs used to clean the network, which is considered to be proportional with the number of infected nodes. The total number of nodes in the network is $N(t)=X(t)+Y(t)+Z(t)$. The significance of each parameter in the model is as follows: $A$ is the inflow rate of susceptible nodes, $\beta$ is the transition rate from $X$ to $Y$ resulting from the contact between susceptible and infected nodes, $d$ is the rate at which nodes are crashed by another cause other than being attacked by viruses, $k$ is the constant rate at which the nodes are being protected by another antivirus software, $\alpha$ is the rate at which infected nodes become incapable of infecting susceptible nodes, $\pi$ is the rate at which infected nodes are recovered and become susceptible again, $\mu$ is the growth rate of antivirus software used to clean the network, $\mu_{0}$ is the rate by which it fails to work efficiently, and $V_{a 0}$ is the number of antivirus programs permanently present in the system to protect the existing software; all these parameters are assumed to be positive.

In real life, the number of contacts of a susceptible node per unit time cannot always increase linearly with $Y$, especially when the number of infectious nodes is large [6], so for more realism, we first suppose that the saturated incidence rate is nonlinear and takes the form $\beta X Y / f(Y)$. Model (1) becomes

$$
\begin{aligned}
& d X=\left(A-\frac{\beta X Y}{f(Y)}-d X-k X+\pi Y V_{a}\right) d t \\
& d Y=\left(\frac{\beta X Y}{f(Y)}-d Y-\alpha Y-\pi Y V_{a}\right) d t \\
& d Z=(k X-d Z) d t \\
& d V_{a}=\left(\mu Y-\mu_{0}\left(V_{a}-V_{a 0}\right)\right) d t
\end{aligned}
$$

where $f$ is a positive function satisfying $f(0)=1$ and $f^{\prime}(Y) \geq$ 0 . Such function $f$ enables the introduction of several effects, like the psychological effects (e.g., $(Y / f(Y)=Y /(1+\gamma Y))$ see [7]). Conforming to the biological epidemic models, this effect is manifested when the number of infected nodes is very large; the infection force may decrease as the number of infections $Y$ increases. In relation to our model, in the presence of a very large number of infectious nodes, the users of computers in the network may tend to reduce the number of contacts of their computers.

In the real world, the parameters of a compartmental model are always subject of random variability that affects the dynamic of the population. There exist several types of noise that may represent the environmental random variability; many studies showed that the white noise is an appropriate representation of environmental random variability in terrestrial systems (see Steele 1985 [10]; Vasseur and Yodzis 2004 [11]). For this reason, we introduce a Gaussian white noise disturbance into this model by considering the case where the parameters $d$ and $\beta$ are subject to random fluctuations. Hence, by using the technique of parameter perturbation, which represents the commonly used procedure in constructing SDE models (e.g., Zhang et al. [4], Ji et al. [12]), we replace $d$ by $d+\sigma_{1} d B_{1}$ and $\beta$ by $\beta+\sigma_{2} d B_{2}$, where $B_{1}(t)$ and $B_{2}(t)$ are standard one-dimensional independent Brownian motions and $\sigma_{1}$ and $\sigma_{2}$ their intensities. The other parameters are the same as in system (1). We obtain the following stochastic system:

$$
\begin{aligned}
d X= & \left(A-\frac{\beta X Y}{f(Y)}-d X-k X+\pi Y V_{a}\right) d t-\sigma_{1} X d B_{1} \\
& -\sigma_{2} \frac{X Y}{f(Y)} d B_{2} \\
d Y= & \left(\frac{\beta X Y}{f(Y)}-d Y-\alpha Y-\pi Y V_{a}\right) d t-\sigma_{1} Y d B_{1} \\
& +\sigma_{2} \frac{X Y}{f(Y)} d B_{2} \\
d Z= & (k X-d Z) d t-\sigma_{1} Z d B_{1} \\
d V_{a}= & \left(\mu Y-\mu_{0}\left(V_{a}-V_{a 0}\right)\right) d t
\end{aligned}
$$

The added value of this paper is summarized in the fact that presents a deterministic and stochastic study of a global model that takes into consideration the effect of antivirus programs in the spread of malware in a computer network. Also, it contains a global nonlinear saturated incidence rate which reflects more realism.

The remainder of this paper is as follows: in Section 2, we present some preliminary results related to the following study. We investigate in Section 3 the stability of the two equilibrium states of the deterministic system (2). In Section 4, we show that system (3) admits a global and unique positive solution, starting from the initial values $\left(X(0), Y(0), Z(0), V_{a}(0)\right)$. Next in Section 5, we give a sufficient condition for the system to be extinct. In Section 6, sufficient condition for the existence of a unique stationary distribution is obtained. We conclude in Section 7 by presenting some numerical simulations to illustrate our results.

\section{Preliminaries}

Throughout the rest of this paper, we let $(\Omega, \mathbb{F}, \mathbb{P})$ be a complete probability space with a filtration $\left(\mathbb{F}_{t}\right)_{t \geq 0}$ satisfying the usual conditions (i.e., it is increasing and right continuous while $\mathbb{F}_{0}$ contains all $\mathbb{P}$-null sets $) . B_{i}(t),(i=1,2)$ are defined on this complete probability space, we also denote

$$
\mathbb{R}_{+}^{4}=\left\{\left(\chi_{1}, \chi_{2}, \chi_{3}, \chi_{4}\right) \chi_{i}>0, i=1,2,3,4\right\} \text {. }
$$

In general, consider the $d$ dimensional stochastic differential equation

$$
\begin{aligned}
d \chi(t)=f(\chi(t), t) d t+g(\chi(t), t) d B(t), & \\
& \text { for } t \geq 0
\end{aligned}
$$

with initial value $\chi(0)=x_{0} \in \mathbb{R}^{d} . B(t)$ denotes an $n$-dimensional standard Brownian motion defined on the complete probability space $\left(\Omega, \mathbb{F},\left\{\mathbb{F}_{t}\right\}_{t \geq 0}, \mathbb{P}\right)$. We denote by $\left(C^{2,1}\left(\mathbb{R}_{d} \times\right.\right.$ $\left.\left[t_{0}, \infty\right], \mathbb{R}_{+}\right)$the family of all nonnegative functions $V(\chi, t)$ 
defined on $\mathbb{R}_{d} \times\left[t_{0}, \infty\right]$ such that they are continuously twice differentiable in $\chi$ and once in $t$. The differential operator $L$ is defined in [13].

Let $X(t)$ be a homogeneous Markov process in $E_{d}\left(E_{d}\right.$ denotes the $d$-dimensional Euclidean space) and be described by the following stochastic differential equation:

$$
d X(t)=b(X) d t+\sum_{r=1}^{k} g_{r}(X) d B_{r}(t) .
$$

The diffusion matrix is defined as follows:

$$
A(\chi)=\left(a_{i j}(\chi)\right), \quad a_{i j}=\sum_{r=1}^{k} g_{r}^{i}(\chi) g_{r}^{j}(\chi) \text {. }
$$

The following lemma is used to prove the theorem related to the stationary distribution for SDE (3) (see [14], Theorem 3.13, p. 1164, Remark 3.2, p. 1160, Theorem 4.3, p. 1168, and Theorem 4.4, p. 1169).

Lemma 1. System (6) is positive recurrent if there is a bounded open subset $U$ of $\mathbb{R}^{n}$ with a regular boundary, and the following holds:

(B1) There exist some $l=1,2, \ldots, n$ and a positive constant $v$ such that

$$
a_{l l}(x) \geq v, \quad \text { for any } x \in U
$$

(B2) There exists a nonnegative function $V: U^{c} \rightarrow \mathbb{R}$ such that $V$ is twice continuously differentiable and that for some $\theta>0$

$$
L V \leq-\theta, \text { for any } x \in U^{c} .
$$

Moreover, the positive recurrent process $X(t)$ has a unique stationary distribution $\pi(\cdot)$ with density in $\mathbb{R}^{n}$ such that for any Borel set $B \in \mathbb{R}^{n}$

$$
\begin{aligned}
& \lim _{t \rightarrow \infty} \mathbb{P}(t, x, B)=\pi(B) \\
& \mathbb{P}_{\chi}\left\{\lim _{T \rightarrow \infty} \frac{1}{T} \int_{0}^{T} f(X(t)) d t=\int_{\mathbb{R}^{n}} f(\chi(t)) \pi(d(\chi))\right\} \\
& \quad=1,
\end{aligned}
$$

for all $\chi \in \mathbb{R}^{n}$, where $f: \mathbb{R}^{n} \rightarrow \mathbb{R}$ is a function integrable with respect to the measure $\pi$.

\section{Equilibrium Analysis and Global Stability of Equilibria}

The basic reproduction number is the number of secondary infectious cases produced by an infectious individual during his or her effective infectious period when introduced in a population of susceptibles [15]. For model (2) the basic reproduction number is

$$
\mathscr{R}_{p}=\frac{\beta A}{(d+k)\left(d+\alpha+\pi V_{a 0}\right)},
$$

where $\mathscr{R}_{p}$ acts as a sharp threshold between extinction and invasion of the disease.
Proposition 2. System (2) admits the virus-free equilibrium state $E^{0}\left(A /(d+k), 0, k A / d(d+k), V_{a 0}\right)$, which exists for all parameters values, and a unique virus-epidemic equilibrium state $E^{*}\left(X^{*}, Y^{*}, Z^{*}, V_{a}^{*}\right)$ which exists if $\mathscr{R}_{p}>1$.

Proof. System (2) has the virus-free equilibrium state $E^{0}\left(A /(d+k), 0, k A / d(d+k), V_{a 0}\right)$; this equilibrium is obtained by giving $Y=0$ (i.e., absence of infection). The positive virus-epidemic equilibrium state is the solution of the following system (equivalent to system (2), except $Y \neq 0$ ):

$$
\begin{aligned}
\beta X-f(Y)\left(d+\alpha+\pi V_{a}\right) & =0, \\
X & =\frac{A-(d+\alpha) Y}{d+k}, \\
Z & =\frac{k}{d}\left(\frac{A-(d+\alpha) Y}{d+k}\right) d t, \\
V_{a} & =\frac{\mu}{\mu_{0}} Y+V_{a 0} .
\end{aligned}
$$

Substituting $X$ and $V_{a}$ by their expression in the first equation of system (12), we get the following equation of $Y$ :

$$
\begin{aligned}
\Lambda(Y)= & \frac{\beta A}{d+k}-\frac{\beta(d+\alpha)}{d+k} Y \\
& -f(Y)\left[d+\alpha+\pi\left(\frac{\mu}{\mu_{0}} Y+V_{a 0}\right)\right]=0,
\end{aligned}
$$

since $f^{\prime} \geq 0$, then $\Lambda$ is decreasing. Moreover, we have

$$
\Lambda(Y)<\frac{\beta A}{d+k}-\frac{\beta(d+\alpha)}{d+k} Y .
$$

Thus

$$
\lim _{Y \rightarrow+\infty} \Lambda(Y)=-\infty
$$

and also, we have $f(0)=1$; then

$$
\begin{aligned}
\Lambda(0) & =\frac{\beta A}{d+k}-\left(d+\alpha+\pi V_{a 0}\right) \\
& =\left(d+\alpha+\pi V_{a 0}\right)\left(\mathscr{R}_{p}-1\right),
\end{aligned}
$$

where $\Lambda$ is decreasing; then (15) and (16) imply that $\Lambda$ has a unique positive zero if and only if $\mathscr{R}_{p}>1$.

The following theorem gives sufficient conditions for the global stability of equilibria of model (2).

Theorem 3. If $\mathscr{R}_{p} \leq 1$, then the virus-free equilibrium state $E^{0}$ is globally asymptotically stable. While if $\mathscr{R}_{p}>1$, then the virus-epidemic equilibrium state $E^{*}$ is globally asymptotically stable. 
Proof. Using the Lyapunov asymptotic theorem [16] to prove the global asymptotic stability of $E^{0}$. Let us consider the function

$$
\begin{aligned}
V_{1}= & \frac{\omega_{1}}{2}\left(Z-Z^{0}\right)^{2}+\frac{\omega_{2}}{2}\left(X+Y-X^{0}\right)^{2} \\
& +\frac{\omega_{3}}{2}\left(V_{a}-V_{a}^{0}\right)^{2}+\omega_{4} \int_{0}^{Y} f(s) d s \\
& +\frac{\omega_{5}}{2}\left(X+Y+Z-X^{0}-Z^{0}\right)^{2}
\end{aligned}
$$

where $E^{0}=\left(X^{0}, Y^{0}, Z^{0}, V_{a}^{0}\right)$ is the virus-free equilibrium state determined in the precedent theorem. $\omega_{1}, \omega_{2}, \omega_{3}, \omega_{4}$, and $\omega_{5}$ are positive constants that will be determined later. We have

$$
\begin{aligned}
V_{1}\left(E^{0}\right) & =0, \\
V_{1}\left(X, Y, Z, V_{a}\right) & >0 \quad \forall\left(X, Y, Z, V_{a}\right) \neq E^{0}, \\
V_{1}(x) & \longrightarrow \infty
\end{aligned}
$$

as $\|x\| \longrightarrow \infty$,

$$
x \in \mathbb{R}^{4} .
$$

The time derivative of $V_{1}$ along solutions of system (2) is

$$
\begin{aligned}
\dot{V}_{1}=\omega_{1} & \left(Z-Z^{0}\right)(k X-d Z)+\omega_{2}\left(X+Y-X^{0}\right) \\
& \cdot(A-(d+k) X-(d+\alpha) Y)+\omega_{3}\left(V_{a}-V_{a 0}\right) \\
& \cdot\left(\mu Y-\mu_{0}\left(V_{a}-V_{a 0}\right)\right)+\omega_{4} f(Y) \\
& \cdot\left(\frac{\beta X Y}{f(Y)}-d Y-\alpha Y-\pi Y V_{a}\right) \\
+ & \omega_{5}\left(X+Y+Z-X^{0}-Z^{0}\right) \\
& \cdot(A-d(X+Y+Z)-\alpha Y) .
\end{aligned}
$$

At $E^{0}$, we have

$$
\begin{aligned}
A-d X^{0}-k X^{0} & =0, \\
k X^{0}-d Z^{0} & =0 .
\end{aligned}
$$

Then

$$
\begin{aligned}
\dot{V}_{1} & =\omega_{1}\left(Z-Z^{0}\right)\left(k\left(X-X^{0}\right)-d\left(Z-Z^{0}\right)\right) \\
& +\omega_{2}\left(X+Y-X^{0}\right)\left(-(d+k)\left(X-X^{0}\right)\right. \\
& -(d+\alpha) Y)+\omega_{3}\left(V_{a}-V_{a 0}\right)\left(\mu Y-\mu_{0}\left(V_{a}-V_{a 0}\right)\right)
\end{aligned}
$$

$$
\begin{aligned}
& +\omega_{4} \beta X Y-\omega_{4} f(Y)\left((d+\alpha) Y+\pi Y V_{a}\right) \\
& +\omega_{5}\left(\left(X-X^{0}\right)+\left(Z-Z^{0}\right)+Y\right)\left(-d\left(X-X^{0}\right)\right. \\
& \left.-d\left(Z-Z^{0}\right)-(d+\alpha) Y\right)=-\omega_{5} d\left(Z-Z^{0}\right)^{2} \\
& -\omega_{3} \mu_{0}\left(V_{a}-V_{a 0}\right)^{2}-\left(\omega_{2}(d+k)+\omega_{5} d\right)(X \\
& \left.-X^{0}\right)^{2}-\omega_{5}(d+\alpha) Y^{2}+\left(\omega_{1} k-2 \omega_{5} d\right)\left(Z-Z^{0}\right) \\
& -\left(X-X^{0}\right)+\left(\omega_{4} \beta-\omega_{2}(2 d+k+\alpha)\right. \\
& \left.-\omega_{5}(2 d+\alpha)\right) Y\left(X-X^{0}\right)-\left[\omega_{1} d\left(Z-Z^{0}\right)^{2}\right. \\
& \left.+\omega_{5}(2 d+\alpha) Y\left(Z-Z^{0}\right)+\omega_{2}(d+\alpha) Y^{2}\right]+\left(\omega_{3} \mu\right. \\
& \left.-\omega_{4} \pi f(Y)\right) Y V_{a}-Y\left(\omega_{3} \mu V_{a 0}+\omega_{4} f(Y)(d+\alpha)\right. \\
& \left.-\beta \omega_{4} X^{0}\right) .
\end{aligned}
$$

Let

$$
\begin{aligned}
& \omega_{1}=\frac{1}{d}, \\
& \omega_{5}=\frac{\omega_{1} k}{2 d}, \\
& \omega_{2}=\left(\frac{\omega_{5}(2 d+\alpha)}{2}\right)^{2} \frac{1}{d+\alpha}, \\
& \omega_{4}=\frac{\omega_{2}(2 d+k+\alpha)+\omega_{5}(2 d+\alpha)}{\beta}, \\
& \omega_{3}=\frac{\omega_{4} \pi}{\mu} .
\end{aligned}
$$

Then

$$
\begin{aligned}
\dot{V}_{1} & =-\omega_{5} d\left(Z-Z^{0}\right)^{2}-\omega_{3} \mu_{0}\left(V_{a}-V_{a 0}\right)^{2} \\
& -\left(\omega_{2}(d+k)+\omega_{5} d\right)\left(X-X^{0}\right)^{2}-\omega_{5}(d+\alpha) Y^{2} \\
& -\left[\left(Z-Z^{0}\right)+2 \frac{\omega_{5}(2 d+\alpha)}{2} Y\left(Z-Z^{0}\right)\right. \\
& \left.+\left(\frac{\omega_{5}(2 d+\alpha)}{2} Y\right)^{2}\right]+\omega_{4} \pi(1-f(Y)) Y V_{a} \\
& -\omega_{4} Y\left(\pi V_{a 0}+f(Y)(d+\alpha)-\beta X^{0}\right) .
\end{aligned}
$$

We have $f(Y(t)) \geq 1$ for all $t \geq 0$; then

$$
\begin{gathered}
\dot{V}_{1} \leq-\omega_{5} d\left(Z-Z^{0}\right)^{2}-\omega_{3} \mu_{0}\left(V_{a}-V_{a 0}\right)^{2} \\
-\left(\omega_{2}(d+k)+\omega_{5} d\right)\left(X-X^{0}\right)^{2}
\end{gathered}
$$




$$
\begin{aligned}
& -\omega_{5}(d+\alpha) Y^{2}-\left(Z-Z^{0}+\frac{\omega_{5}(2 d+\alpha)}{2} Y\right)^{2} \\
& -\omega_{4} Y\left(\pi V_{a 0}+d+\alpha-\beta \frac{A}{d+k}\right) .
\end{aligned}
$$

Thus

$$
\begin{aligned}
\dot{V}_{1} \leq & -\omega_{5} d\left(Z-Z^{0}\right)^{2}-\omega_{3} \mu_{0}\left(V_{a}-V_{a 0}\right)^{2} \\
& -\left(\omega_{2}(d+k)+\omega_{5} d\right)\left(X-X^{0}\right)^{2} \\
& -\omega_{5}(d+\alpha) Y^{2} \\
& -\omega_{4}\left(d+\alpha+\pi V_{a 0}\right) Y\left(1-\mathscr{R}_{p}\right) .
\end{aligned}
$$

Thus, if $\mathscr{R}_{p} \leq 1$ then $\dot{V}_{1}<0$ for all $\left(X, Y, Z, V_{a}\right) \neq E^{0}$. Hence by Lyapunov asymptotic theorem [16], $E^{0}$ is globally asymptotically stable.

For the stability of the virus-epidemic equilibrium state $E^{*}$, we refer the reader to Remark 13 in Section 6.

Remark 4. Theorem 3 showed that the basic reproductive number $\mathscr{R}_{p}$ acts as a sharp threshold determining when the disease (i.e., spread of computer viruses) becomes endemic for model (2). Furthermore, the disease is permanent with simple dynamics whenever $\mathscr{R}_{p}>1$.

\section{Existence and Uniqueness of the Global Nonnegative Solution}

The solution $\left(X(t), Y(t), Z(t), V_{a}(t)\right)$ of (3) presents the sizes of susceptible nodes, infected nodes, protected nodes, and antivirus programs used to clean the network at time $t$, respectively. Then, they should all be nonnegative. For this reason, and to study the dynamical behavior of system (3), the first concern is whether the solution is of global and positive existence.

Theorem 5. Let $\left(X(0), Y(0), Z(0), V_{a}(0)\right) \in \mathbb{R}_{+}^{4}$; then there is a unique positive solution to SDE (3) on $t \geq 0$; this solution remains in $\mathbb{R}_{+}^{4}$ with probability 1 .

Proof. Since the coefficients of system (3) are locally Lipschitz continuous, for any given initial value $(X(0), Y(0)$, $\left.Z(0), V_{a}(0)\right) \in \mathbb{R}_{+}^{4}$ then, there is a unique local solution $\left(X(t), Y(t), Z(t), V_{a}(t)\right)$ on $t \in\left[0, \tau_{e}\right)$, where $\tau_{e}$ is the explosion time [13]. We show next that $\tau_{e}=+\infty$ almost surely. For this purpose, we define the stopping time

$$
\begin{aligned}
\tau_{\infty} & =\inf \left\{t \in\left[0, \tau_{e}\right): X(t) \leq 0 \text { or } Y(t) \leq 0 \text { or } Z(t)\right. \\
& \left.\leq 0 \text { or } V_{a}(t) \leq 0\right\}
\end{aligned}
$$

Throughout this paper we set inf $\emptyset=\infty$, where $\emptyset$ denotes the empty set. One can see that $\tau_{\infty} \leq \tau_{e}$, if we prove that $\tau_{\infty}=$ $\infty$ a.s.; then, $\tau_{e}=\infty$ a.s.; thus $\left(X(t), Y(t), Z(t), V_{a}(t)\right) \in \mathbb{R}_{+}^{4}$ a.s. If this statement is false, then there is a pair of constants
$T>0$ and $\epsilon \in(0,1)$ such that $P\left(\tau_{\infty} \leq T\right) \geq \epsilon$. We define a $C^{2}$-function $V$ for $\left(X(t), Y(t), Z(t), V_{a}(t)\right) \in \mathbb{R}_{+}^{4}$ by $V\left(X, Y, Z, V_{a}\right)=\ln X Y Z V_{a}$. Setting $\Omega=\left(\tau_{\infty} \leq T\right)$ and using Itô's formula we get, for all $\omega \in \Omega$ and $t \in\left[0, \tau_{\infty}\right)$,

$$
\begin{aligned}
d V\left(X, Y, Z, V_{a}\right)= & L V\left(X, Y, Z, V_{a}\right) d t-3 \sigma_{1} d B_{1} \\
& +\sigma_{2}\left(\frac{X-Y}{f(Y)}\right) d B_{2}
\end{aligned}
$$

where

$$
\begin{aligned}
& L V\left(X, Y, Z, V_{a}\right)=\left(\frac{1}{X}\right)\left(A-\frac{\beta X Y}{f(Y)}-d X-k X\right. \\
& \left.+\pi Y V_{a}\right)-\frac{\sigma_{1}^{2}}{2}-\frac{\sigma_{2}^{2} Y^{2}}{2 f^{2}(Y)}+\left(\frac{1}{Y}\right)\left(\frac{\beta X Y}{f(Y)}-d Y\right. \\
& \left.-\alpha Y-\pi Y V_{a}\right)-\frac{\sigma_{1}^{2}}{2}-\frac{\sigma_{2}^{2} X^{2}}{2 f^{2}(Y)}+\left(\frac{1}{Z}\right)(k X \\
& -d Z)-\frac{\sigma_{1}^{2}}{2}+\left(\frac{1}{V_{a}}\right)\left(\mu Y-\mu_{0}\left(V_{a}-V_{a 0}\right)\right) \\
& \geq\left(-3 d-k-\alpha-\mu_{0}-\frac{3 \sigma_{1}^{2}}{2}-\frac{\beta Y}{f(Y)}\right. \\
& \left.-\frac{\sigma_{2}^{2}}{2}\left(\frac{Y^{2}+X^{2}}{f^{2}(Y)}\right)-\pi V_{a}\right) .
\end{aligned}
$$

We know that $f$ is a positive function that verifies $f(0)=$ 1 and $f^{\prime}(Y) \geq 0$ for all $Y>0$; then

$$
\begin{aligned}
& L V\left(X, Y, Z, V_{a}\right) \geq\left(-3 d-k-\alpha-\mu_{0}-\frac{3 \sigma_{1}^{2}}{2}-\beta Y\right. \\
& \left.\quad-\frac{\sigma_{2}^{2}}{2}\left(Y^{2}+X^{2}\right)-\pi V_{a}\right) .
\end{aligned}
$$

Therefore, we get

$$
\begin{aligned}
d V\left(X, Y, Z, V_{a}\right) \geq & h\left(X, Y, V_{a}\right) d t-3 \sigma_{1} d B_{1} \\
& +\sigma_{2}\left(\frac{X-Y}{f(Y)}\right) d B_{2},
\end{aligned}
$$

where

$$
\begin{aligned}
& h\left(X, Y, V_{a}\right)=\left(-3 d-k-\alpha-\mu_{0}-\frac{3 \sigma_{1}^{2}}{2}-\beta Y\right. \\
& \left.\quad-\frac{\sigma_{2}^{2}}{2}\left(Y^{2}+X^{2}\right)-\pi V_{a}\right) .
\end{aligned}
$$


Thereafter, we get

$$
\begin{aligned}
V\left(X, Y, Z, V_{a}\right) \geq & V\left(X(0), Y(0), Z(0), V_{a}(0)\right) \\
& +\int_{0}^{t} h\left(X(s), Y(s), V_{a}(s)\right) d s \\
& -3 \sigma_{1} B_{1}(t) \\
& +\int_{0}^{t} \sigma_{2}\left(\frac{X(s)-Y(s)}{f(Y(s))}\right) d B_{2}(s) .
\end{aligned}
$$

Note that, for $\omega \in \Omega$, there is at least one of $X\left(\tau_{\infty}, \omega\right)$, $Y\left(\tau_{\infty}, \omega\right), Z\left(\tau_{\infty}, \omega\right)$ and $V_{a}\left(\tau_{\infty}, \omega\right)$ that equal 0 . Thereby

$$
\lim _{t \rightarrow \tau_{\infty}} V\left(X(t), Y(t), Z(t), V_{a}(t)\right)=-\infty
$$

Letting $t \rightarrow \infty$, in (32), leads to the contradiction that

$$
\begin{aligned}
-\infty \geq & V\left(X(0), Y(0), Z(0), V_{a}(0)\right) \\
& +\int_{0}^{\tau_{\infty}} h\left(X(s), Y(s), V_{a}(s)\right) d s-3 \sigma_{1} B_{1}\left(\tau_{\infty}\right) \\
& +\int_{0}^{\tau_{\infty}} \sigma_{2}\left(\frac{X-Y}{f(Y)}\right) d B_{2}(s)>-\infty
\end{aligned}
$$

So $\tau_{\infty}=\infty$ a.s. This completes the proof of Theorem 5 .

\section{Extinction}

One of the main interests of epidemiology is how to control the disease dynamics so that it will be eliminated in a long term. In this section, we shall establish sufficient conditions for the extinction of viruses in the stochastic model of computer network alimented by a system of antivirus software (3).

Lemma 6. Let $\left(X(t), Y(t), Z(t), V_{a}(t)\right)$ be the solution of system (3). Then with any initial value $\left(X(0), Y(0), Z(0), V_{a}(0)\right) \in$ $\mathbb{R}_{+}^{4}$, we have

$$
\lim _{t \rightarrow \infty} \frac{X(t)+Y(t)}{t}=0 \quad \text { a.s. }
$$

Moreover

$$
\begin{aligned}
& \lim _{t \rightarrow \infty} \frac{X(t)}{t}=0 \quad \text { a.s., } \\
& \lim _{t \rightarrow \infty} \frac{Y(t)}{t}=0 \quad \text { a.s. }
\end{aligned}
$$

Proof. Let $v(t)=X(t)+Y(t)$ and $\Phi(v)=(1+v)^{p}$, where $p>0$ will be chosen later. Using Itô's formula, we get

$$
\begin{aligned}
d \Phi(v(t))= & L \Phi(v) d t \\
& +p(1+v)^{p-1}\left(-\sigma_{1} X d B_{1}-\sigma_{1} Y d B_{1}\right),
\end{aligned}
$$

where

$$
\begin{aligned}
& L \Phi(v)=p(1+v)^{p-1}[A-d v-k X-\alpha Y] \\
& +\frac{p(p-1)}{2}(1+v)^{p-2} \sigma_{1}^{2} v^{2}=p(1+v)^{p-2} \\
& \quad \cdot\left\{(1+v)(A-d v-k X-\alpha Y)+\frac{(p-1)}{2} \sigma_{1}^{2} v^{2}\right\} \\
& \quad \leq p(1+v)^{p-2}\left\{(1+v)(A-d v)+\frac{(p-1)}{2} \sigma_{1}^{2} v^{2}\right\} \\
& \quad=p(1+v)^{p-2} \\
& \quad \cdot\left\{-\left[d-\frac{(p-1)}{2} \sigma_{1}^{2}\right] v^{2}+(A-d) v+A\right\} .
\end{aligned}
$$

Let $p>0$ such that $d-((p-1) / 2) \sigma_{1}^{2}:=\lambda>0$; then

$$
L \Phi(v) \leq p(1+v)^{p-2}\left\{-\lambda v^{2}+(A-d) v+A\right\} ，
$$

so

$$
\begin{gathered}
d \Phi(v) \leq p(1+v)^{p-2}\left\{-\lambda v^{2}+(A-d) v+A\right\} d t \\
-p \sigma_{1} v(1+v)^{p-1} d B_{1},
\end{gathered}
$$

and let $0<k<p \lambda$; we have

$$
\begin{aligned}
d\left(e^{k t} \Phi(v(t))\right)= & L\left(e^{k t} \Phi(v)\right) d t \\
& -p \sigma_{1} e^{k t} v(1+v)^{p-1} d B_{1},
\end{aligned}
$$

and thus

$$
E\left(e^{k t} \Phi(v(t))\right)=\Phi(v(0))+E \int_{0}^{t} L\left(e^{k s} \Phi(v(s))\right) d s,
$$

where

$$
\begin{aligned}
& L\left(e^{k t} \Phi(v(t))\right)=k e^{k t} \Phi(v(t))+e^{k t} L \Phi(v(t)) \\
& \quad \leq p e^{k t}(1+v)^{p-2} \\
& \quad \cdot\left\{\frac{k}{p}(1+v)^{2}-\lambda v^{2}+(A-d) v+A\right\} \\
& =p e^{k t}(1+v)^{p-2} \\
& \quad \cdot\left\{-\left(\lambda-\frac{k}{p}\right) v^{2}+\left(A-d+\frac{2 k}{p}\right) v+A+\frac{k}{p}\right\} \\
& \leq p e^{k t} C ;
\end{aligned}
$$

here

$$
\begin{aligned}
C= & \sup _{v \in \mathbb{R}_{+}}\left\{(1+v)^{p-2}\right. \\
& \left.\cdot\left(-\left(\lambda-\frac{k}{p}\right) v^{2}+\left(A-d+\frac{2 k}{p}\right) v+A+\frac{k}{p}\right)\right\} ;
\end{aligned}
$$


thus from (42)

$$
E\left(e^{k t}(1+v)^{p}\right) \leq(1+v(0))^{p}+\frac{p C}{k} e^{k t}
$$

therefore

$$
\limsup _{t \rightarrow \infty} E\left((1+v)^{p}\right) \leq \frac{p C}{k}=: C_{0}, \quad \text { a.s.; }
$$

in addition to this result, $v$ is continuous, which implies that there exists a constant $M>0$, such that

$$
E\left((1+v(t))^{p}\right) \leq M, \quad t \geq 0,
$$

with (47), we can proceed as in [8] to complete the proof.

Lemma 7. For any initial value $\left(X(0), Y(0), Z(0), V_{a}(0)\right) \in$ $\mathbb{R}_{+}^{4}$, the solution $\left(X(t), Y(t), Z(t), V_{a}(t)\right)$ of system (3) verifies

$$
\begin{aligned}
\lim _{t \rightarrow \infty} \frac{\int_{0}^{t} X(s) d B_{1}(s)}{t} & =0, \quad \text { a.s. } \\
\lim _{t \rightarrow \infty} \frac{\int_{0}^{t} Y(s) d B_{1}(s)}{t} & =0, \quad \text { a.s. } \\
\lim _{t \rightarrow \infty} \frac{\int_{0}^{t}(X(s) / f(Y(s))) d B_{2}(s)}{t} & =0 \quad \text { a.s. }
\end{aligned}
$$

Proof. Let $M_{1}=\int_{0}^{t} X(s) / f(Y(s)) d B_{2}(s)$ and $p>2$. By using the Burkholder-Davis-Gundy inequality [13] and (47), we have

$$
\begin{aligned}
E\left[\sup _{0 \leq s \leq t}\left|M_{1}(s)\right|^{p}\right] & \leq C_{p} E\left[\int_{0}^{t} \frac{X^{2}(s)}{f^{2}(Y(s))} d s\right]^{p / 2} \\
& \leq C_{p} t^{p / 2} E\left[\sup _{0 \leq s \leq t} \frac{X^{p}(s)}{f^{p}(Y(s))}\right] \\
& \leq M_{p} C_{p} t^{p / 2} .
\end{aligned}
$$

Let $\epsilon$ be an arbitrary positive constant; then according to Chebyshev's inequality we have

$$
\begin{gathered}
P\left\{\omega: \sup _{k \delta \leq t \leq(k+1) \delta}\left|M_{1}(t)\right|^{p}>(k \delta)^{1+\epsilon+p / 2}\right\} \\
\leq \frac{M_{p} C_{p}((k+1) \delta)^{p / 2}}{(k \delta)^{1+\epsilon+p / 2}} \leq \frac{2^{p / 2} M_{p} C_{p}}{(k \delta)^{1+\epsilon}},
\end{gathered}
$$

an application of Doob's martingale inequality, and the BorelCantelli lemma [13] gives, for almost all $\omega \in \Omega$ and for all except finitely many $k$,

$$
\sup _{k \delta \leq t \leq(k+1) \delta}\left|M_{1}(t)\right|^{p} \leq(k \delta)^{1+\epsilon+p / 2},
$$

and then, for almost $\omega \in \Omega$, there exists an integer $k_{0}(\omega)$ such that, for all $k \geq k_{0}$, (51) holds. Thus, if $k \geq k_{0}$ and $k \delta \leq t \leq$ $(k+1) \delta$, we get

$$
\frac{\ln \left|M_{1}(t)\right|^{p}}{\ln t} \leq \frac{(1+\epsilon+p / 2) \ln (k \delta)}{(\ln k \delta)} \leq 1+\epsilon+\frac{p}{2},
$$

\section{Consequently}

$$
\limsup _{t \rightarrow+\infty} \frac{\ln \left|M_{1}(t)\right|}{\ln t} \leq \frac{1+\epsilon+p / 2}{p}, \text { a.s. }
$$

and by letting $\epsilon \rightarrow 0$, we obtain

$$
\limsup _{t \rightarrow+\infty} \frac{\ln \left|M_{1}(t)\right|}{\ln t} \leq \frac{1}{p}+\frac{1}{2}, \quad \text { a.s.; }
$$

therefore, for arbitrary small constant $\delta$, such that $0<\delta<$ $1 / 2-1 / p$, there exists a constant $K(\omega)$ and a set $\Omega_{\delta}$, such that $P\left(\Omega_{\delta}\right) \geq 1-\delta$ and for all $t \geq K(\omega), \omega \in \Omega_{\delta}$

$$
\ln \left|M_{1}(t)\right| \leq\left(\frac{1}{2}+\frac{1}{p}+\delta\right) \ln t
$$

thus

$$
\limsup _{t \rightarrow+\infty} \frac{M_{1}(t)}{t} \leq \limsup _{t \rightarrow+\infty} \frac{t^{1 / 2+1 / p+\delta}}{t}=0, \text { a.s, }
$$

together with $\lim \inf _{t \rightarrow+\infty}\left|M_{1}(t)\right| / t \geq 0$, a.s., yields

$$
\lim _{t \rightarrow+\infty} \frac{\left|M_{1}(t)\right|}{t}=0 \text {, a.s. }
$$

So

$$
\lim _{t \rightarrow+\infty} \frac{M_{1}(t)}{t}=\lim _{t \rightarrow+\infty} \frac{\int_{0}^{t} X(s) / f(Y(s)) d B_{2}(s)}{t}=0,
$$
that

Taking $f=1$ and using the same arguments, we can show

$$
\begin{aligned}
& \lim _{t \rightarrow \infty} \frac{\int_{0}^{t} X(s) d B_{1}(s)}{t}=0, \quad \text { a.s. } \\
& \lim _{t \rightarrow \infty} \frac{\int_{0}^{t} Y(s) d B_{1}(s)}{t}=0, \quad \text { a.s. }
\end{aligned}
$$

This completes the proof of Lemma 7.

Theorem 8. Let $\left(X(t), Y(t), Z(t), V_{a}(t)\right)$ be the solution of system (3) with any positive initial value $\left(X(0), Y(0), Z(0), V_{a}(0)\right)$; then

$$
\lim _{t \rightarrow \infty} \frac{\ln Y}{t} \leq-\left(d+\alpha+\frac{\sigma_{1}^{2}}{2}-\frac{\beta^{2}}{2 \sigma_{2}^{2}}\right), \quad \text { a.s. }
$$

Assume that $\beta^{2}<2 \sigma_{2}^{2}\left(d+\alpha+\sigma_{1}^{2} / 2\right)$; then $Y(t)$ tends to zero exponentially almost surely (i.e., the disease dies out with probability one).

Proof. By application of Itô's formula to system (3) we get

$d \ln Y(t)$

$$
\begin{aligned}
= & \left(\frac{\beta X}{f(Y)}-d-\alpha-\pi V_{a}-\frac{\sigma_{1}^{2}}{2}-\frac{\sigma_{2}^{2} X^{2}}{2 f^{2}(Y)}\right) d t \\
& -\sigma_{1} d B_{1}+\sigma_{2} \frac{X}{f(Y)} d B_{2},
\end{aligned}
$$


and then

$$
\begin{aligned}
\ln Y \leq & \ln Y(0) \\
& +\int_{0}^{t}\left(\frac{\beta X(s)}{f(Y(s))}-\frac{\sigma_{2}^{2} X^{2}(s)}{2 f^{2}(Y(s))}-\pi V_{a}(s)\right) d s \\
& -\left(d+\alpha+\frac{\sigma_{1}^{2}}{2}\right) t-\sigma_{1} B_{1}(t)+\sigma_{2} M_{1}(t),
\end{aligned}
$$

where

$$
M_{1}(t)=\int_{0}^{t} \frac{X(s)}{f(Y(s))} d B_{2}(s)
$$

then

$$
\begin{aligned}
\ln Y(t) \leq & \ln Y(0)-\frac{\sigma_{2}^{2}}{2} \int_{0}^{t}\left(\frac{X(s)}{f(Y(s))}-\frac{\beta}{\sigma_{2}^{2}}\right)^{2} d s \\
& +\frac{\beta^{2}}{2 \sigma_{2}^{2}} t-\left(d+\alpha+\frac{\sigma_{1}^{2}}{2}\right) t-\sigma_{1} B_{1}(t) \\
& +\sigma_{2} M_{1}(t) \\
\leq & \ln Y(0)-\left(d+\alpha+\frac{\sigma_{1}^{2}}{2}-\frac{\beta^{2}}{2 \sigma_{2}^{2}}\right) t \\
& -\sigma_{1} B_{1}(t)+\sigma_{2} M_{1}(t) ;
\end{aligned}
$$

therefore, by dividing both sides of (64) by $t$, we obtain

$$
\begin{aligned}
\frac{\ln Y}{t} \leq & \frac{\ln Y(0)}{t}-\left(d+\alpha+\frac{\sigma_{1}^{2}}{2}-\frac{\beta^{2}}{2 \sigma_{2}^{2}}\right)-\frac{\sigma_{1} B_{1}(t)}{t} \\
& +\frac{\sigma_{2} M_{1}(t)}{t} .
\end{aligned}
$$

Using the strong law of large numbers [13], we obtain $\lim _{t \rightarrow \infty}\left(\sigma_{1} B_{1}(t) / t\right)=0$, a.s., and by (Lemma 7), we have

$$
\lim _{t \rightarrow \infty} \frac{\sigma_{2} M_{1}(t)}{t}=0, \quad \text { a.s., }
$$

taking the limit superior of both sides of (65) and the condition $\beta^{2}<2 \sigma_{2}^{2}\left(d+\alpha+\sigma_{1}^{2} / 2\right)$ leads to

$$
\limsup _{t \rightarrow \infty} \frac{\ln Y}{t} \leq-\left(d+\alpha+\frac{\sigma_{1}^{2}}{2}-\frac{\beta^{2}}{2 \sigma_{2}^{2}}\right)<0, \quad \text { a.s.; }
$$

thus $\lim _{t \rightarrow \infty} Y(t)=0$, a.s. This completes the proof of Theorem 8 .

Remark 9. Theorem 8 shows that the number of infected nodes $Y(t)$ goes to extinction almost surely, where $\beta^{2}<$ $2 \sigma_{2}^{2}\left(d+\alpha+\sigma_{1}^{2} / 2\right)$. Namely, large white noise stochastic disturbances conduct to control the infection of nodes.

\section{Stationary Distribution and Positive Recurrence}

The following theorem investigates the stability in a stochastic sense; it gives a sufficient condition for the existence of an asymptotically invariant distribution for the solution of model (3). The proof is based on Lemma 1 and uses the Lyapunov function method. However, there exist some other methods to investigate the stationary distribution, like the method based on the Markov semigroups theory (see Rudnicki and Pichór [17], Lin et al. [18]). Furthermore, some other authors have given the explicit expression of the density of the stationary distribution through solving the Fokker Planck equation of their proposed model (see Buonocore et al. [19], $\mathrm{Xu}[20])$.

Theorem 10. Consider the stochastic system (3) with positive initial value; suppose that $\mathscr{R}_{p}>1$ and

$$
0<\gamma<\min \left(\gamma_{1} X^{*^{2}}, \gamma_{2} Y^{*^{2}}, \gamma_{3} Z^{*^{2}}, \gamma_{4} V_{a}^{*^{2}}\right),
$$

where

$$
\begin{aligned}
\gamma_{1}= & a_{2}\left(d+k-2 \sigma_{1}^{2}\right)+a_{5}\left(d-4 \sigma_{1}^{2}\right)-a_{4} \sigma_{2}^{2} Y^{*}, \\
\gamma_{2}= & a_{5}\left(d+\alpha-4 \sigma_{1}^{2}\right)-2 a_{2} \sigma_{1}^{2}, \\
\gamma_{3}= & a_{5}\left(d-4 \sigma_{1}^{2}\right)-a_{1} \sigma_{1}^{2}, \\
\gamma_{4}= & a_{3} \mu_{0}, \\
\gamma= & 2 a_{2} \sigma_{1}^{2}\left(X^{*^{2}}+Y^{*^{2}}\right)+a_{1} \sigma_{1}^{2} Z^{*^{2}}+a_{4} \sigma_{2}^{2} Y^{*} X^{*^{2}} \\
& +a_{4} \frac{\sigma_{1}^{2} Y^{*}}{2}+4 a_{5} \sigma_{1}^{2}\left(X^{*^{2}}+Y^{*^{2}}+Z^{*^{2}}\right), \\
a_{1}= & \frac{1}{d}, \\
a_{5}= & \frac{k}{2 d^{2}}, \\
a_{2}= & \frac{(2 d+\alpha)^{2}}{4(d+\alpha)} a_{5}^{2}, \\
a_{4}= & \frac{\left(a_{5}(2 d+\alpha)+a_{2}(\alpha+2 d+k)\right) f\left(Y^{*}\right)}{\beta}, \\
a_{3}= & \frac{\pi}{\mu} a_{4} .
\end{aligned}
$$

Then the solution $\left(X, Y, Z, V_{a}\right)$ is positive recurrent and admits a unique ergodic stationary distribution.

Remark 11. One can see that condition (68) can be verified for sufficiently small environmental noises $\sigma_{1}$ and $\sigma_{2}$. In fact, we have

$$
\begin{aligned}
& \lim _{\sigma_{1}, \sigma_{2} \rightarrow 0} \gamma_{1}=a_{2}(d+k)+a_{5} d>0, \\
& \lim _{\sigma_{1}, \sigma_{2} \rightarrow 0} \gamma_{2}=a_{5}(d+\alpha)>0, \\
& \lim _{\sigma_{1}, \sigma_{2} \rightarrow 0} \gamma_{3}=a_{5} d>0,
\end{aligned}
$$

while

$$
\lim _{\sigma_{1}, \sigma_{2} \rightarrow 0} \gamma=0
$$


Proof. We consider the Lyapunov function $V$ defined for $\left(X, Y, Z, V_{a}\right) \in \mathbb{R}_{+}^{4}$ by

$$
\begin{aligned}
V\left(X, Y, Z, V_{a}\right)= & a_{1} V_{1}(Z)+a_{2} V_{2}(X, Y)+a_{3} V_{3}\left(V_{a}\right) \\
& +a_{4} V_{4}(Y)+a_{5} V_{5}(X, Y, Z),
\end{aligned}
$$

where $V_{1}, V_{2}, V_{3}, V_{4}$, and $V_{5}$ are positive functions defined for $\left(X, Y, Z, V_{a}\right) \in \mathbb{R}_{+}^{4}$ by

$$
\begin{aligned}
V_{1}(Z) & =\frac{1}{2}\left(Z-Z^{*}\right)^{2}, \\
V_{2}(X, Y) & =\frac{1}{2}\left(X+Y-X^{*}-Y^{*}\right)^{2}, \\
V_{3}\left(V_{a}\right) & =\frac{1}{2}\left(V_{a}-V_{a}^{*}\right)^{2}, \\
V_{4}(Y) & =Y-Y^{*}-Y^{*} \ln \frac{Y}{Y^{*}}, \\
V_{5}(X, Y, Z) & =\frac{1}{2}\left(X+Y+Z-X^{*}-Y^{*}-Z^{*}\right)^{2},
\end{aligned}
$$

and $a_{1}, a_{2}, a_{3}, a_{4}$, and $a_{5}$ are well defined in (70). By applying the differential operator $L$ to $V_{1}$, we obtain

$$
L V_{1}(Z)=\left(Z-Z^{*}\right)(k X-d Z)+\frac{\sigma_{1}^{2}}{2} Z^{2}
$$

At the equilibrium state $E^{*}\left(X^{*}, Y^{*}, Z^{*}, V_{a}^{*}\right)$, we get $k X^{*}-$ $d Z^{*}=0$; then

$$
\begin{aligned}
L V_{1}(Z)= & \left(Z-Z^{*}\right)\left(k\left(X-X^{*}\right)-d\left(Z-Z^{*}\right)\right) \\
& +\frac{\sigma_{1}^{2}}{2}\left(\left(Z-Z^{*}\right)+Z^{*}\right)^{2} .
\end{aligned}
$$

An application of the inequality $(x+y)^{2} \leq 2\left(x^{2}+y^{2}\right)$ for all $(x, y) \in \mathbb{R}^{2}$ yields

$$
\begin{aligned}
L V_{1}(Z) \leq & -\left(d-\sigma_{1}^{2}\right)\left(Z-Z^{*}\right)^{2} \\
& +k\left(X-X^{*}\right)\left(Z-Z^{*}\right)+\sigma_{1}^{2} Z^{*^{2}} .
\end{aligned}
$$

For the function $V_{2}$, we have

$$
\begin{aligned}
& L V_{2}(X, Y) \\
& =\left(X+Y-X^{*}-Y^{*}\right)(A-d X-k X-d Y-\alpha Y) \\
& \quad+\frac{\sigma_{1}^{2}}{2}(X+Y)^{2} ;
\end{aligned}
$$

at the equilibrium state $E^{*}$ we have $A-d X^{*}-k X^{*}-d Y^{*}-$ $\alpha Y^{*}=0$; hence

$$
\begin{aligned}
& L V_{2}(X, Y) \leq\left(X+Y-X^{*}-Y^{*}\right) \\
& \cdot\left(-(d+k)\left(X-X^{*}\right)-(d+\alpha)\left(Y-Y^{*}\right)\right) \\
& \quad+\sigma_{1}^{2}\left(X^{2}+Y^{2}\right) \leq-(d+k)\left(X-X^{*}\right)^{2}-(d+\alpha) \\
& \cdot\left(Y-Y^{*}\right)^{2}-(2 d+k+\alpha)\left(X-X^{*}\right)\left(Y-Y^{*}\right) \\
& \quad+\sigma_{1}^{2}\left(\left(X-X^{*}\right)+X^{*}\right)^{2}+\sigma_{1}^{2}\left(\left(Y-Y^{*}\right)+Y^{*}\right)^{2} .
\end{aligned}
$$

Therefore

$$
\begin{aligned}
L V_{2}(X, Y) \leq & -\left(d+k-2 \sigma_{1}^{2}\right)\left(X-X^{*}\right)^{2} \\
& -\left(d+\alpha-2 \sigma_{1}^{2}\right)\left(Y-Y^{*}\right)^{2} \\
& -(2 d+k+\alpha)\left(X-X^{*}\right)\left(Y-Y^{*}\right) \\
& +2 \sigma_{1}^{2} X^{*^{2}}+2 \sigma_{1}^{2} Y^{*^{2}} .
\end{aligned}
$$

Now, we calculate $L V_{3}$

$$
L V_{3}\left(V_{a}\right)=\left(V_{a}-V_{a}^{*}\right)\left(\mu Y-\mu_{0}\left(V_{a}-V_{a 0}\right)\right) .
$$
0 ; then

At the equilibrium state $E^{*}$, we have $\mu Y^{*}-\mu_{0}\left(V_{a}^{*}-V_{a 0}\right)=$

$$
L V_{3}\left(V_{a}\right)=\left(V_{a}-V_{a}^{*}\right)\left(\mu\left(Y-Y^{*}\right)-\mu_{0}\left(V_{a}-V_{a}^{*}\right)\right) ;
$$
thus

$$
L V_{3}\left(V_{a}\right)=-\mu_{0}\left(V_{a}-V_{a}^{*}\right)^{2}+\mu\left(V_{a}-V_{a}^{*}\right)\left(Y-Y^{*}\right) .
$$

For $L V_{4}$, we have

$$
\begin{aligned}
L V_{4}(Y)= & \left(1-\frac{Y^{*}}{Y}\right)\left(\frac{\beta X Y}{f(Y)}-d Y-\alpha Y-\pi Y V_{a}\right) \\
& +\frac{Y^{*}}{2}\left(\sigma_{1}^{2}+\sigma_{2}^{2} \frac{X^{2}}{f^{2}(Y)}\right) \\
\leq & \left(Y-Y^{*}\right)\left(\frac{\beta X}{f(Y)}-d-\alpha-\pi V_{a}\right) \\
& +\frac{Y^{*}}{2}\left(\sigma_{1}^{2}+\sigma_{2}^{2} \frac{X^{2}}{f^{2}(Y)}\right) ;
\end{aligned}
$$

we have at the equilibrium state $E^{*}, \beta X^{*} / f\left(Y^{*}\right)-d-\alpha-\pi V_{a}^{*}=$ 0 ; hence

$$
\begin{aligned}
& L V_{4}(Y) \\
& \leq\left(Y-Y^{*}\right)\left(\frac{\beta X}{f(Y)}-\frac{\beta X^{*}}{f\left(Y^{*}\right)}-\pi\left(V_{a}-V_{a}^{*}\right)\right) \\
& \quad+\frac{Y^{*}}{2}\left(\sigma_{1}^{2}+\sigma_{2}^{2} \frac{X^{2}}{f^{2}(Y)}\right)
\end{aligned}
$$

the function $f$ is chosen to verify that $f$ is nondecreasing and $f(Y) \geq 1$; thus

$$
\begin{aligned}
& L V_{4}(Y) \leq\left(Y-Y^{*}\right)\left(\beta X\left(\frac{1}{f(Y)}-\frac{1}{f\left(Y^{*}\right)}\right)\right. \\
& \left.+\frac{\beta}{f\left(Y^{*}\right)}\left(X-X^{*}\right)-\pi\left(V_{a}-V_{a}^{*}\right)\right)+\frac{Y^{*}}{2}\left(\sigma_{1}^{2}\right. \\
& \left.+\sigma_{2}^{2} \frac{X^{2}}{f^{2}(Y)}\right) \leq\left(Y-Y^{*}\right)\left(\frac{\beta}{f\left(Y^{*}\right)}\left(X-X^{*}\right)\right. \\
& \left.-\pi\left(V_{a}-V_{a}^{*}\right)\right)+Y^{*} \sigma_{2}^{2}\left(X-X^{*}\right)^{2}+\sigma_{2}^{2} Y^{*} X^{*^{2}} \\
& +\frac{\sigma_{1}^{2} Y^{*}}{2} .
\end{aligned}
$$


Thus

$$
\begin{aligned}
L V_{4}(Y) \leq & Y^{*} \sigma_{2}^{2}\left(X-X^{*}\right)^{2} \\
& +\frac{\beta}{f\left(Y^{*}\right)}\left(X-X^{*}\right)\left(Y-Y^{*}\right) \\
& -\pi\left(V_{a}-V_{a}^{*}\right)\left(Y-Y^{*}\right)+\sigma_{2}^{2} Y^{*} X^{*^{2}} \\
& +\frac{\sigma_{1}^{2} Y^{*}}{2} .
\end{aligned}
$$

Next, we calculate $L V_{5}$

$$
\begin{aligned}
L V_{5}(X, Y, Z)= & \left(X-X^{*}+Y-Y^{*}+Z-Z^{*}\right) \\
& \cdot(A-d X-d Y-\alpha Y-d Z) \\
& +\frac{\sigma_{1}^{2}}{2}(X+Y+Z)^{2} .
\end{aligned}
$$

At the equilibrium state $E^{*}$ we have $A-d X^{*}-d Y^{*}-\alpha Y^{*}-$ $d Z^{*}=0$. Using the inequality $(x+y+z)^{2} \leq 4\left(x^{2}+y^{2}+z^{2}\right)$ for all $(x, y, z) \in \mathbb{R}^{3}$ we get

$$
\begin{aligned}
& L V_{5}(X, Y, Z) \leq\left(X-X^{*}+Y-Y^{*}+Z-Z^{*}\right) \\
& \cdot\left(-d\left(X-X^{*}\right)-(d+\alpha)\left(Y-Y^{*}\right)-d\left(Z-Z^{*}\right)\right) \\
& +2 \sigma_{1}^{2}\left(X^{2}+Y^{2}+Z^{2}\right) \leq-d\left(X-X^{*}\right)^{2}-(d+\alpha) \\
& \cdot\left(Y-Y^{*}\right)^{2}-d\left(Z-Z^{*}\right)^{2}-(2 d+\alpha)\left(X-X^{*}\right) \\
& \cdot\left(Y-Y^{*}\right)-2 d\left(X-X^{*}\right)\left(Z-Z^{*}\right)-(2 d+\alpha) \\
& \cdot\left(Y-Y^{*}\right)\left(Z-Z^{*}\right) \\
& +4 \sigma_{1}^{2}\left(\left(X-X^{*}\right)^{2}+\left(Y-Y^{*}\right)^{2}+\left(Z-Z^{*}\right)^{2}\right) \\
& +4 \sigma_{1}^{2}\left(X^{*^{2}}+Y^{*^{2}}+Z^{*^{2}}\right) .
\end{aligned}
$$

So

$$
\begin{aligned}
L V_{5}(X, Y, Z) \leq & -\left(d-4 \sigma_{1}^{2}\right)\left(X-X^{*}\right)^{2} \\
& -\left(d+\alpha-4 \sigma_{1}^{2}\right)\left(Y-Y^{*}\right)^{2} \\
& -\left(d-4 \sigma_{1}^{2}\right)\left(Z-Z^{*}\right)^{2} \\
& -(2 d+\alpha)\left(X-X^{*}\right)\left(Y-Y^{*}\right) \\
& -2 d\left(X-X^{*}\right)\left(Z-Z^{*}\right) \\
& -(2 d+\alpha)\left(Y-Y^{*}\right)\left(Z-Z^{*}\right) \\
& +4 \sigma_{1}^{2}\left(X^{*^{2}}+Y^{*^{2}}+Z^{*^{2}}\right) .
\end{aligned}
$$

Combining (77), (80), (83), (87), and (90) and multiplying by coefficients $a_{1}, a_{2}, a_{3}, a_{4}$, and $a_{5}$ of (73) determined in (70), we get

$$
\begin{aligned}
& L V\left(X, Y, Z, V_{a}\right) \leq-\gamma_{1}\left(X-X^{*}\right)^{2}-\gamma_{2}\left(Y-Y^{*}\right)^{2} \\
& -\gamma_{3}\left(Z-Z^{*}\right)^{2}-\gamma_{4}\left(V-V_{a}^{*}\right)^{2}-\gamma_{5}\left(X-X^{*}\right)(Y \\
& \left.-Y^{*}\right)-\gamma_{6}\left(X-X^{*}\right)\left(Z-Z^{*}\right)-\gamma_{7}\left(V-V_{a}^{*}\right)(Y \\
& \left.-Y^{*}\right)-\left[a_{1} d\left(Z-Z^{*}\right)^{2}+a_{2}(d+\alpha)\left(Y-Y^{*}\right)^{2}\right. \\
& \left.+a_{5}(2 d+\alpha)\left(Y-Y^{*}\right)\left(Z-Z^{*}\right)\right]+\gamma,
\end{aligned}
$$

where

$$
\begin{aligned}
& \gamma_{5}=a_{5}(2 d+\alpha)+a_{2}(\alpha+2 d+k)-a_{4} \frac{\beta}{f\left(Y^{*}\right)}, \\
& \gamma_{6}=2 a_{5} d-k a_{1}, \\
& \gamma_{7}=-\mu a_{3}+a_{4} \pi,
\end{aligned}
$$

by substituting $a_{1}, a_{2}, a_{3}, a_{4}$, and $a_{5}$ into $\gamma_{5}, \gamma_{6}$, and $\gamma_{7}$, We can see that $\gamma_{5}=\gamma_{6}=\gamma_{7}=0$; thus

$$
\begin{aligned}
L V( & \left.X, Y, Z, V_{a}\right) \\
\leq & -\gamma_{1}\left(X-X^{*}\right)^{2}-\gamma_{2}\left(Y-Y^{*}\right)^{2}-\gamma_{3}\left(Z-Z^{*}\right)^{2} \\
& -\gamma_{4}\left(V-V_{a}^{*}\right)^{2} \\
& -\left(\left(Z-Z^{*}\right)+a_{5} \frac{2 d+\alpha}{2}\left(Y-Y^{*}\right)\right)^{2}+\gamma .
\end{aligned}
$$

As a result

$$
\begin{aligned}
L V\left(X, Y, Z, V_{a}\right) \leq & -\gamma_{1}\left(X-X^{*}\right)^{2}-\gamma_{2}\left(Y-Y^{*}\right)^{2} \\
& -\gamma_{3}\left(Z-Z^{*}\right)^{2}-\gamma_{4}\left(V-V_{a}^{*}\right)^{2} \\
& +\gamma,
\end{aligned}
$$

as well, by letting condition (68) hold, and considering a positive constant $\gamma^{\prime}$ such that

$$
0<\gamma<\gamma^{\prime}<\min \left(\gamma_{1} X^{*^{2}}, \gamma_{2} Y^{*^{2}}, \gamma_{3} Z^{*^{2}}, \gamma_{4} V_{a}^{*^{2}}\right),
$$

we have

$$
\begin{aligned}
L V\left(X, Y, Z, V_{a}\right) \leq & -\gamma_{1}\left(X-X^{*}\right)^{2}-\gamma_{2}\left(Y-Y^{*}\right)^{2} \\
& -\gamma_{3}\left(Z-Z^{*}\right)^{2}-\gamma_{4}\left(V-V_{a}^{*}\right)^{2} \\
& +\gamma^{\prime}-\left(\gamma^{\prime}-\gamma\right) ;
\end{aligned}
$$

the ellipsoid

$$
\begin{aligned}
& -\gamma_{1}\left(X-X^{*}\right)^{2}-\gamma_{2}\left(Y-Y^{*}\right)^{2}-\gamma_{3}\left(Z-Z^{*}\right)^{2} \\
& -\gamma_{4}\left(V-V_{a}^{*}\right)^{2}+\gamma^{\prime},
\end{aligned}
$$




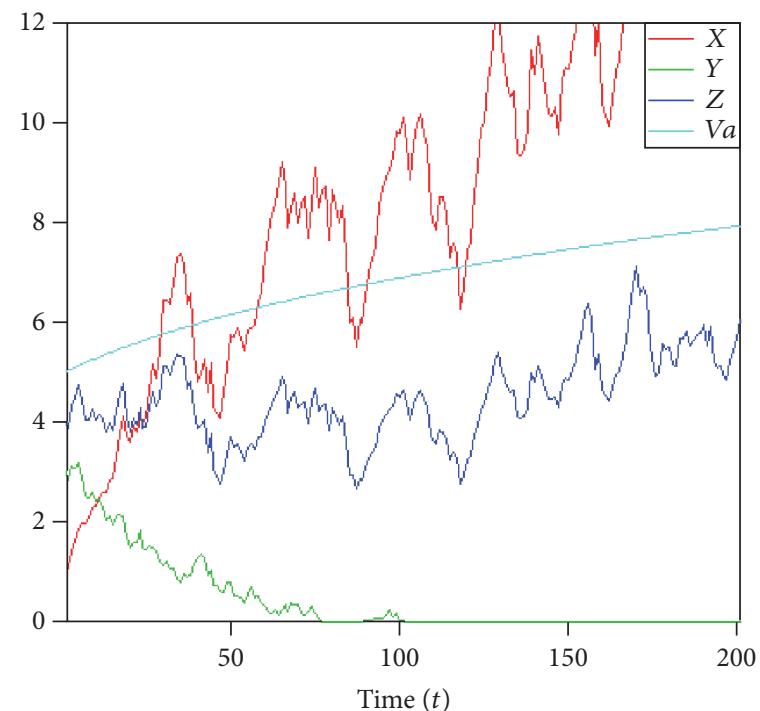

(a) The stochastic system

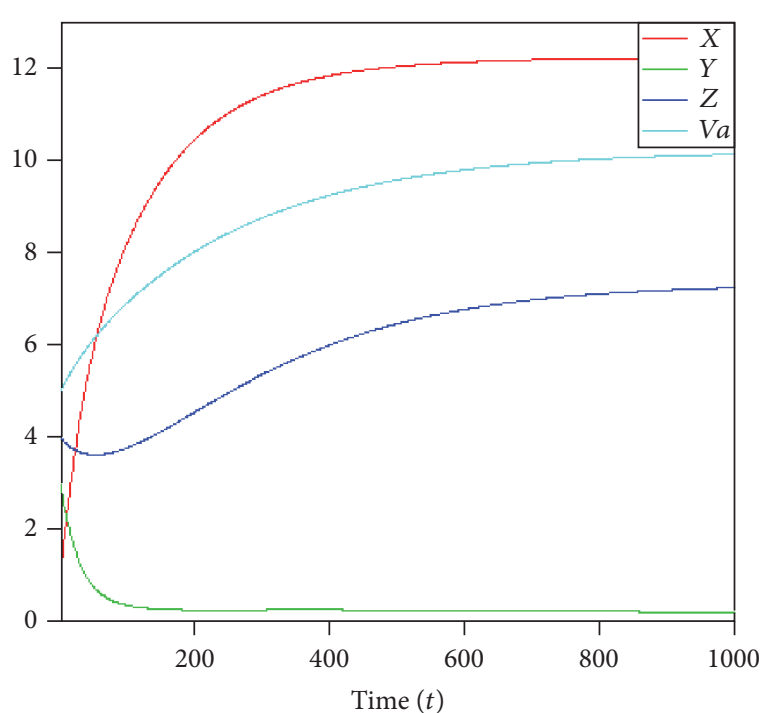

(b) The deterministic system

Figure 1: Computer simulation of the path $X(t), Y(t), Z(t), V_{a}(t)$ for models (a) (3) and (b) (2), for parameters of Example 14, and $\left(X(0), Y(0), Z(0), V_{a}(0)\right)=(2,4,3,5)$.

lies entirely in $\mathbb{R}_{+}^{4}$. We can take as $U$ any neighborhood of the ellipsoid such that $\bar{U} \subset \mathbb{R}_{+}^{4}$, where $\bar{U}$ is closer to $U$. Thus, $L V\left(X, Y, Z, V_{a}\right) \leq-\left(\gamma^{\prime}-\gamma\right)$ for any $\left(X, Y, Z, V_{a}\right) \in \mathbb{R}_{+}^{4} \backslash U$; this implies that condition (B2) of Lemma 1 is satisfied. Condition (B1) of the same lemma remains to be verified. The diffusion matrix associated with system (3) is given by

$$
\begin{aligned}
& M(X, Y, Z) \\
& =\left[\begin{array}{cccc}
\sigma_{1}^{2} X^{2}+\sigma_{2}^{2} \frac{X^{2} Y^{2}}{f^{2}(Y)} & \sigma_{1}^{2} X Y-\sigma_{2}^{2} \frac{X^{2} Y^{2}}{f^{2}(Y)} & \sigma_{1}^{2} X Z & 0 \\
\sigma_{1}^{2} X Y-\sigma_{2}^{2} \frac{X^{2} Y^{2}}{f^{2}(Y)} & \sigma_{1}^{2} Y^{2}+\sigma_{2}^{2} \frac{X^{2} Y^{2}}{f^{2}(Y)} & \sigma_{1}^{2} Y Z & 0 \\
\sigma_{1}^{2} X Z & \sigma_{1}^{2} Y Z & \sigma_{1}^{2} Z^{2} & 0 \\
0 & 0 & 0 & 0
\end{array}\right] .
\end{aligned}
$$

Since $U$ is a bounded open subset that lies entirely in $\mathbb{R}_{+}^{4}$, then for all $\left(X, Y, Z, V_{a}\right) \in U$

$$
a_{33}\left(X, Y, Z, V_{a}\right)=\sigma_{1}^{2} Z^{2} \geq \min _{\left(X, Y, Z, V_{a}\right) \in \bar{U}} \sigma_{1}^{2} Z^{2}>v
$$

where $v$ is a positive constant; condition (B1) is met. Consequently we have shown conditions (B1) and (B2) of Lemma 1.

Remark 12. Theorem 10 treats the long time behavior of (3). In the case $\mathscr{R}_{p}>1$, and for sufficiently small environment noises, after some initial transients the solutions $X(t), Y(t)$, $V(t)$, and $V_{a}(t)$ fluctuate around the deterministic steadystate values $X^{*}, Y^{*}, Z^{*}$, and $V_{a}^{*}$, respectively.

Remark 13. By taking $\sigma_{1}=\sigma_{2}=0$ and the same function (73) with the same parameters defined in (70), if $\mathscr{R}_{p}>1$, one can easily follow the same proof below (B2) to show that

$$
\begin{aligned}
\dot{V}\left(X, Y, Z, V_{a}\right) \leq & -\gamma_{1}\left(X-X^{*}\right)^{2}-\gamma_{2}\left(Y-Y^{*}\right)^{2} \\
& -\gamma_{3}\left(Z-Z^{*}\right)^{2}-\gamma_{4}\left(V-V_{a}^{*}\right)^{2} .
\end{aligned}
$$

Then $\dot{V}<0$ for all $\left(X, Y, Z, V_{a}\right) \neq E^{*}$. Moreover, $V\left(E^{*}\right)=$ 0 and $V\left(X, Y, Z, V_{a}\right)>0$ for all $\left(X, Y, Z, V_{a}\right) \neq E^{*}$ and $V(x) \rightarrow$ $\infty$ as $\|x\| \rightarrow \infty$. Then, by Lyapunov asymptotic stability theorem [16], the virus-epidemic equilibrium state $E^{*}$ is globally asymptotically stable.

\section{Discussions and Numerical Simulations}

In this section, we present some numerical simulations to support our theoretical results. Throughout the following numerical simulations, we choose $f(Y)=1+2 Y^{2}$.

We use Milstein's higher-order method [21, 22] to derive the corresponding discretization equations of model (3).

Example 14. We choose the parameters in systems (2) and (3) as follows: $A=10, \beta=0.45, d=0.5, k=0.3, \pi=0.4, \alpha=0.4$, $\mu=0.35, \mu_{0}=0.4, V_{a 0}=10, \sigma_{1}=0.7$, and $\sigma_{2}=0.8$.

Using the above parameters we have

$\mathscr{R}_{p}=1.15>1$, which implies from Theorem 3 that model (2) stabilizes at the virus-epidemic equilibrium state $E^{*}$. Figure 1(b) illustrates this case.

$\beta^{2}=0.2<2 \sigma_{2}^{2}\left(d+\alpha+\sigma_{1}^{2} / 2\right)=1.47,\left(\lim _{t \rightarrow \infty}((\ln Y) / t) \leq\right.$ -0.99 a.s.). Theorem 8 shows that the disease dies out under large white noise disturbances $\sigma_{1}$ and $\sigma_{2}$. The numerical simulation in Figure 1(a) supports this result; $Y(t)$ represented by the green color tends to zero almost surely. 

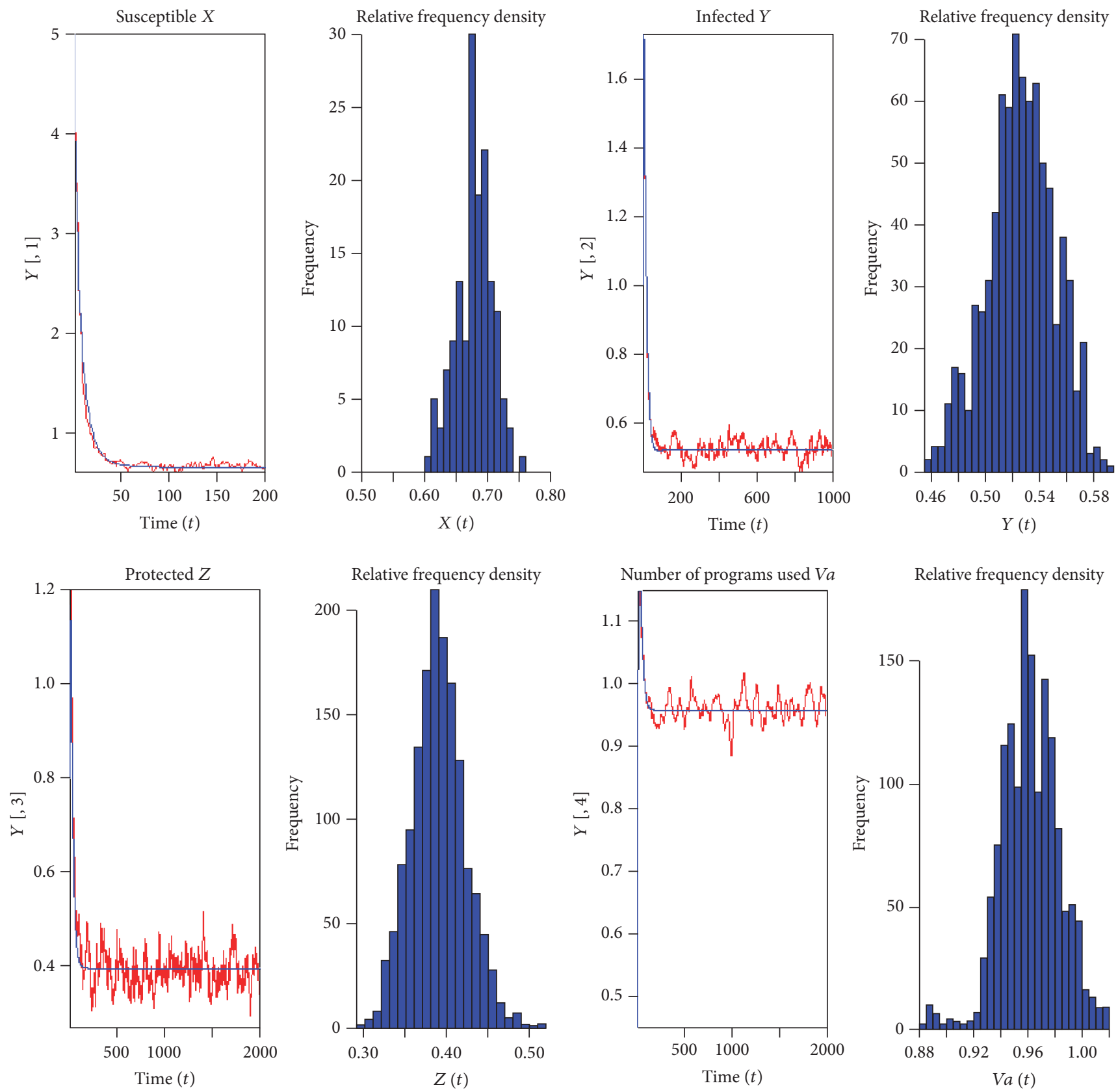

FIGURE 2: The solution of stochastic model (3) and its histograms with parameters in Example 15 and initial values $\left(X(0), Y(0), Z(0), V_{a}(0)\right)=$ $(5,1,0.8,0.45)$.

Example 15. We choose the parameters in systems (2) and (3) as follows: $A=1, \beta=3, d=0.5, k=0.3, \pi=0.4, \alpha=0.4$, $\mu=0.35, \mu_{0}=0.4, V_{a 0}=0.5, \sigma_{1}=0.08$, and $\sigma_{2}=0.08$.

The parameters in Example 15 are chosen to verify the conditions of Theorem 10. Using these parameters, we have $\mathscr{R}_{p}=3.41>1$ and the intensities $\sigma_{1}$ and $\sigma_{2}$ are small enough. Thus, there exists a unique ergodic stationary distribution for system (3). The following simulations illustrate this result.

The red lines represent the densities of each stochastic variable $X(t), Y(t), Z(t)$, and $V_{a}(t)$, respectively, while the blue ones represent the densities of each deterministic variable. Based on these lines and their corresponding histograms, we can observe the existence of a stationary distribution. The result implies that small intensities of the Brownian motions will strengthen the stability of the system.

\section{Conclusion}

In the real world, problems are not deterministic, including stochastic effects into a model that may give more accuracy to investigate and study phenomena. In this paper, we have studied the stability of a deterministic model powered by a system of antivirus programs and with general nonlinear 
incidence rate. We have next considered a stochastic version of this system with noises introduced in the rate at which nodes are crashed due to reasons other than the attacks of viruses $d$ and the transition rate $\beta$. We first proved the existence and positivity of a unique global solution of this model. Then, with this system of antivirus programs, we investigate the extinction of viruses, we have proved that, under large noise intensities $\sigma_{1}$ and $\sigma_{2}$, the number of infected nodes $Y(t)$ tends to zero exponentially almost surely, and thus the disease dies out with probability one; Figure 1(a) illustrates this case. We have also proved that system (3) has the ergodic property as $\mathscr{R}_{p}>1$ and the fluctuations are very small. Numerical simulations in Figure 2 are carried out to illustrate this theoretical result.

Also, it is interesting to investigate the stochastic persistence in mean. Furthermore, we may study a stochastic version of (3) including Markovian switching into all parameters [23]. These studies are in progress.

\section{Conflicts of Interest}

The authors declare that there are no conflicts of interest regarding the publication of this paper.

\section{References}

[1] B. K. Mishra and N. Jha, "SEIQRS model for the transmission of malicious objects in computer network," Applied Mathematical Modelling, vol. 34, no. 3, pp. 710-715, 2010.

[2] H. Yuan and G. Chen, "Network virus-epidemic model with the point-to-group information propagation," Applied Mathematics and Computation, vol. 206, no. 1, pp. 357-367, 2008.

[3] J. Shukla, G. Singh, P. Shukla, and A. Tripathi, "Modeling and analysis of the effects of antivirus software on an infected computer network," Applied Mathematics and Computation, vol. 227, pp. 11-18, 2014.

[4] C. Zhang, Y. Zhao, Y. Wu, and S. Deng, "A stochastic dynamic model of computer viruses," Discrete Dynamics in Nature and Society, vol. 2012, Article ID 264874, 16 pages, 2012.

[5] J. Amador, "The stochastic SIRA model for computer viruses," Applied Mathematics and Computation, vol. 232, pp. 1112-1124, 2014.

[6] V. Capasso and G. Serio, "A generalization of the KermackMcKendrick deterministic epidemic model," Mathematical Biosciences, vol. 42, no. 1-2, pp. 43-61, 2008.

[7] D. Xiao and S. Ruan, "Global analysis of an epidemic model with nonmonotone incidence rate," Mathematical Biosciences, vol. 208, no. 2, pp. 419-429, 2007.

[8] Y. Zhao and D. Jiang, "The threshold of a stochastic SIS epidemic model with vaccination," Applied Mathematics and Computation, vol. 243, pp. 718-727, 2014.

[9] M. E. J. Newman, S. Forrest, and J. Balthrop, "Email networks and the spread of computer viruses," Physical Review E, vol. 66, no. 3, Article ID 035101, 4 pages, 2002.

[10] J. H. Steele, "A comparison of terrestrial and marine ecological systems," Nature, vol. 313, no. 6001, pp. 355-358, 1985.

[11] D. A. Vasseur and P. Yodzis, "The color of environmental noise," Ecology, vol. 85, no. 4, pp. 1146-1152, 2004.
[12] C. Y. Ji, D. Q. Jiang, and N. Z. Shi, "Multigroup SIR epidemic model with stochastic perturbation," Physica A, vol. 390, no. 10, pp. 1747-1762, 2011.

[13] X. Mao, Stochastic Differential Equations and Applications, Horwood, Chichester, UK, 2nd edition, 1997.

[14] C. Zhu and G. Yin, "Asymptotic properties of hybrid diffusion systems," SIAM Journal on Control and Optimization, vol. 49, no. 4, pp. 1155-1179, 2007.

[15] O. Diekmann, J. A. P. Heesterbeek, and J. A. J. Metz, "On the definition and computation of the basic reproductive ratio $R_{0}$ in model for infectious diseases in heterogeneous population," Journal of Mathematical Biology, vol. 28, no. 4, pp. 365-382, 1990.

[16] P. Al Hokayem and E. Gallestey, "Lyapunov stability theory," Nonlinear Systems and Control-Spring, 2015.

[17] R. Rudnicki and K. Pichór, "Influence of stochastic perturbation on prey-predator systems," Mathematical Biosciences, vol. 206, no. 1, pp. 108-119, 2007.

[18] Y. Lin, D. Jiang, and M. Jin, "Stationary distribution of a stochastic SIR model with saturated incidence and its asymptotic stability," Acta Mathematica Scientia. Series B. English Edition, vol. 35, no. 3, pp. 619-629, 2015.

[19] A. Buonocore, L. Caputo, E. Pirozzi, and A. G. Nobile, "A nonautonomous stochastic predator-prey model," Mathematical Biosciences and Engineering, vol. 11, no. 2, pp. 167-188, 2016.

[20] C. Xu, "Global threshold dynamics of a stochastic differential equation SIS model," Journal of Mathematical Analysis and Applications, vol. 447, no. 2, pp. 736-757, 2017.

[21] M. Carletti, K. Burrage, and P. M. Burrage, "Numerical simulation of stochastic ordinary differential equations in biomathematical modelling," Mathematics and Computers in Simulation, vol. 64, no. 2, pp. 271-277, 2004.

[22] D. J. Higham, "An algorithmic introduction to numerical simulation of stochastic differential equations," SIAM Review, vol. 43, no. 3, pp. 525-546, 2001.

[23] A. Gray, H. L. Greenhalgh, X. R. Mao, and J. Pan, "A stochastic differential equation SIS epidemic model," SIAM Journal on Applied Mathematics, vol. 71, no. 3, pp. 876-902, 2011. 


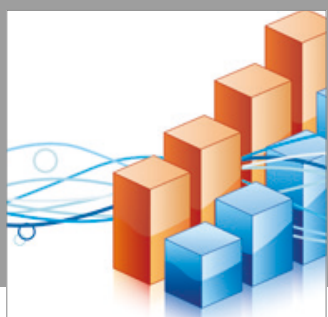

Advances in

Operations Research

vatersals

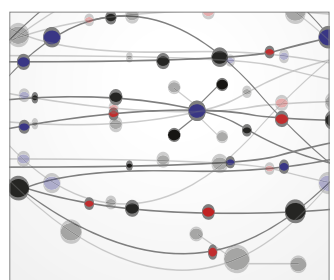

\section{The Scientific} World Journal
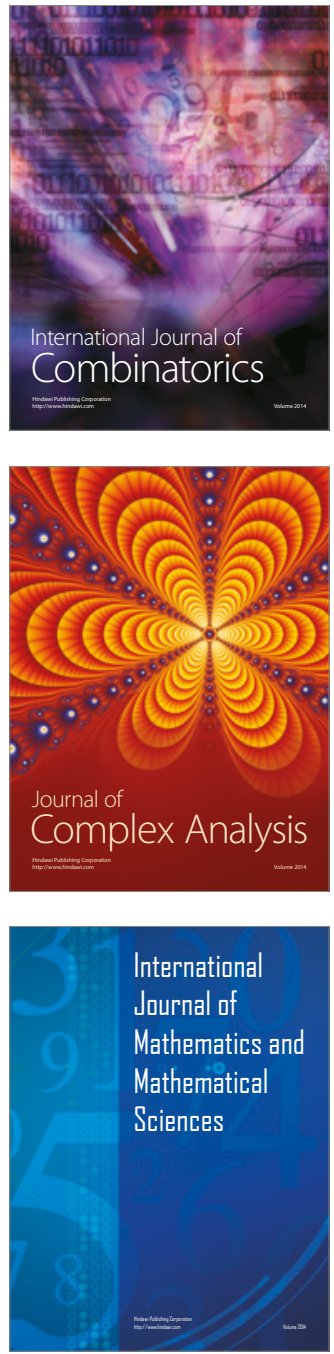
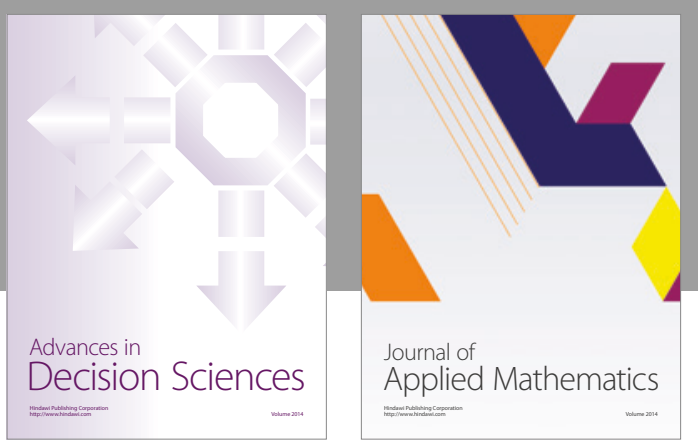

Algebra

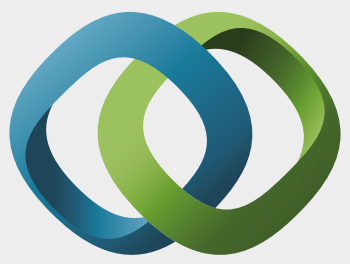

\section{Hindawi}

Submit your manuscripts at

https://www.hindawi.com
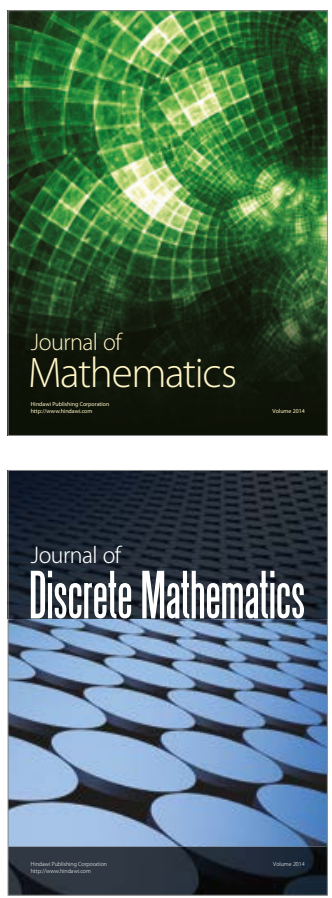

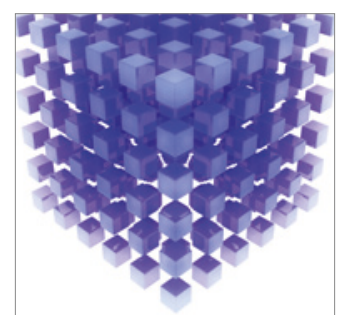

Mathematical Problems in Engineering
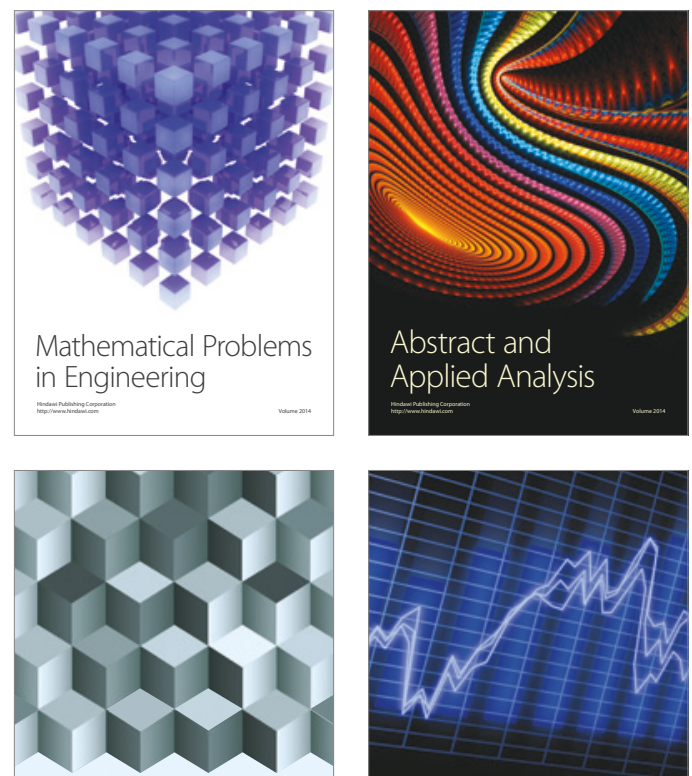

Journal of

Function Spaces

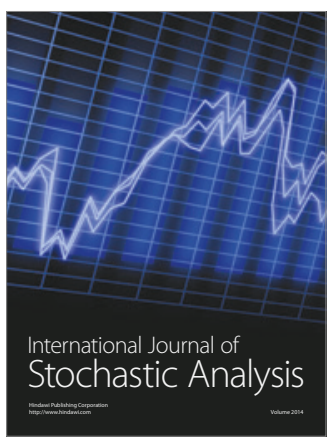

Probability and Statistics
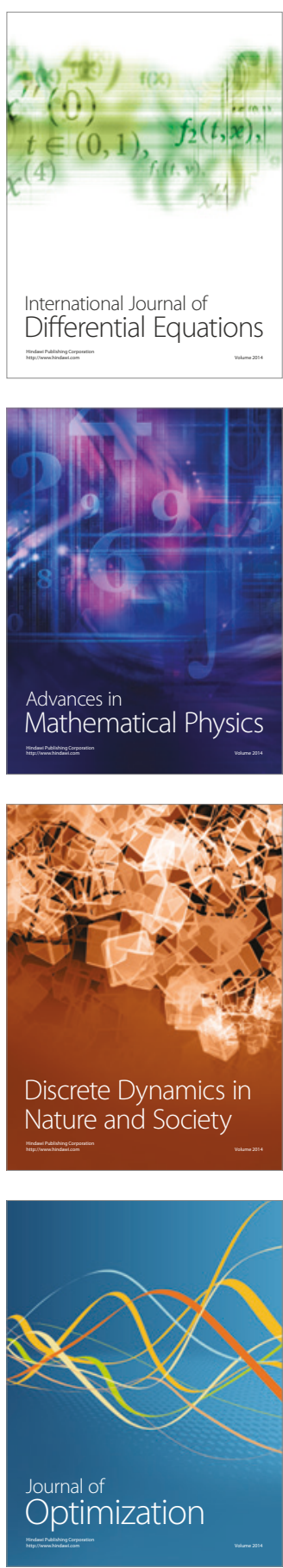